\title{
Creating patient-specific anatomical models for 3D printing and AR/VR: a supplement for the 2018 Radiological Society of North America (RSNA) hands-on course
}

\author{
Nicole Wake ${ }^{1,2^{*}}$ D, Amy E. Alexander ${ }^{3}$, Andy M. Christensen ${ }^{4}$, Peter C. Liacouras ${ }^{5}$, Maureen Schickel ${ }^{6}$, \\ Todd Pietila ${ }^{6}$ and Jane Matsumoto ${ }^{3}$
}

\begin{abstract}
Advanced visualization of medical image data in the form of three-dimensional (3D) printing continues to expand in clinical settings and many hospitals have started to adapt 3D technologies to aid in patient care. It is imperative that radiologists and other medical professionals understand the multi-step process of converting medical imaging data to digital files. To educate health care professionals about the steps required to prepare DICOM data for 3D printing anatomical models, hands-on courses have been delivered at the Radiological Society of North America (RSNA) annual meeting since 2014. In this paper, a supplement to the RSNA 2018 hands-on 3D printing course, we review methods to create cranio-maxillofacial (CMF), orthopedic, and renal cancer models which can be 3D printed or visualized in augmented reality (AR) or virtual reality (VR).
\end{abstract}

Keywords: 3D printing, Virtual reality, Augmented reality

\section{Introduction}

Advanced medical image data visualization in the form of three-dimensional (3D) printing continues to expand in clinical settings. Many hospitals have started to adapt 3D technology to aid in patient care, for use in medical student education, and for research applications. 3D printing originated in the 1980s and encompasses various processes intended to generate a physical model from a digital file [1-3]. Virtual Reality (VR) uses a computer to simulate an alternate 3D environment and allows for user interaction within this space. Augmented Reality (AR), which overlays 3D content in the users real environment, is another method of advanced image visualization that has great potential to transform how physicians access medical imaging data. 3D printed models and AR/VR experiences are expected to provide improvements in the

\footnotetext{
* Correspondence: nwake@montefiore.org

${ }^{1}$ Department of Radiology, Montefiore Medical Center, Albert Einstein College of Medicine, 111 East 210th Street, Bronx, NY 10467, USA

${ }^{2}$ Center for Advanced Imaging Innovation and Research (CAI2R) and Bernard and Irene Schwartz Center for Biomedical Imaging, Department of Radiology, NYU Langone Health, NYU School of Medicine, New York, NY, USA Full list of author information is available at the end of the article
}

visualization of medical images as compared to viewing medical images on a two-dimensional screen [4].

At this time, digital imaging and communications in medicine (DICOM) files cannot be used directly for 3D printing anatomical models. In order to generate patient-specific models for 3D printing and AR/VR, anatomical structures are segmented from DICOM data and the generated structures are converted to virtual 3D models. Next, these files must be saved in a format that is recognized by the 3D printer or AR/VR device. The most common file-type for 3D printing is the stereolithography file format, which is also known as the Standard Tessellation Language or Standard Triangle Language (denoted by the file extension ".stl") and the wavefront or object (.obj) file type, which has the ability to include material properties such as color and shading, is most widely used for AR/VR applications [5].

In order to efficiently create 3D printed anatomic models and to use them safely for medical purposes, radiologists and medical professionals must understand the process of converting medical imaging data to digital files. Therefore, to educate radiologists and other medical professionals about the steps required to prepare 
DICOM data for 3D printing, hands-on courses have been taught at the Radiological Society of North America (RSNA) annual meeting since 2014. Our initial medical 3D printing guide was published for the 2015 RSNA annual meeting [6]. Since then, other guides have been published $[7,8]$ and there remains great interest regarding the many applications of medical 3D printing.

The RSNA 3D Printing Special Interest Group (SIG) has provided published recommendations regarding medical 3D printing [9]. Recommendations have undergone voting during a SIG business meeting by the active membership [9], including a position statement reflecting the use of United States Food and Drug Administration (FDA) cleared software to translate medical images into formats amenable to 3D printing for all aspects of patient care, defined by the SIG as all interactions with healthcare professionals, or patients and their families, related to medical care [8]. This course is educational and does not promote any product. In keeping with SIG recommendations, for the purposes of education we primarily focus on FDA-cleared software for the design and fabrication of patient-specific 3D models. The examples presented in this course include craniomaxillofacial (CMF), orthopedic, and renal cases.

The software used to create "Diagnostic use" anatomical models is considered by the FDA as a class II medical device. At the time the course was delivered, Mimics inPrint (Materialise, Leuven, Belgium) was the only software product with FDA clearance to create 3D printed anatomic models for diagnostic use. While details regarding FDA clearance are beyond the scope of this article, Mimics inPrint was cleared for craniomaxillofacial, cardiovascular, and orthopedic applications [10]. Regarding updates and questions, readers are encouraged to visit the FDA website or consult with the FDA for matters specific to medical 3D printing in the United States [11].

Cranio-maxillofacial 3D printing dates back to the late 1980s [12-14]. It is used today for the management of complex head and neck surgery, craniofacial surgery, endoscopic sinus surgery, and orthognathic surgery helping to ensure the correct resection of margins and repositioning of segments [15-20]. 3D printing in orthopedics dates back to the late 1990s [21], with current applications including upper extremity trauma, deformity, and arthroplasty; foot and ankle surgery; spine surgery; hip and acetabulum surgery; hip and knee arthroplasty; and orthopedic oncology [22-26]. 3D printing soft tissue structures such as the kidneys is relatively new, dating back only a few years [27-29]. 3D printed kidney cancer models can influence pre-surgical planning decisions, which may allow for enhanced performance of minimally invasive organ-sparing procedures [30].

Advanced imaging technologies such as 3D printing, $\mathrm{AR}$, and VR have rapidly been gaining momentum in the medical field. There are many applications of advanced $3 \mathrm{D}$ technologies in medicine including preoperative planning, procedure rehearsal, educational tools for teaching, and patient communication. Herein we review methods to create CMF, orthopedic, and renal cancer models which can be 3D printed or visualized in AR/VR. The ultimate goal is to educate participants about the steps required to create $3 \mathrm{D}$ anatomical models suitable for $3 \mathrm{D}$ printing, AR, or VR from DICOM images.

\section{Workflow}

In general, the steps required for 3D anatomical modeling from DICOM data include the steps shown in Table 1. If imaging is performed with the intent to create an anatomic 3D model, the image acquisition parameters should be optimized for quality [31]. However, this remains challenging considering that imaging studies are typically performed before a model is ordered. Factors to consider include spatial resolution (approximately 1 $\mathrm{mm}^{3}$ ), reconstruction kernel, multi-phase contrast, metal artifact reduction, and sequence parameters for magnetic resonance imaging (MRI). Repeat imaging solely for the purposes of producing a 3D model is often not advisable because it is not cost-efficient and will increase patient radiation dose if a computed tomography $(\mathrm{CT})$ scan is performed.

Image segmentation and post-processing is performed with Mimics inPrint (Materialise NV, Leuven, Belgium). Mimics technology is widely used in academics, hospitals, and the medical device industry for 3D printing [32]. The Mimics inPrint software environment allows for a user friendly workflow to create anatomic regions of interest (ROIs) from the DICOM data and to convert the segmented imaging data to file types that can be used for 3D printing or AR/VR. The workflow consists of five steps including 1) Create ROI, 2) Edit ROI, 3) Add Part, 4) Edit Part, and 5) Prepare Print (Fig. 1). Here, each ROI is one segmented anatomical region and

Table 1 Stages of the anatomical modeling process

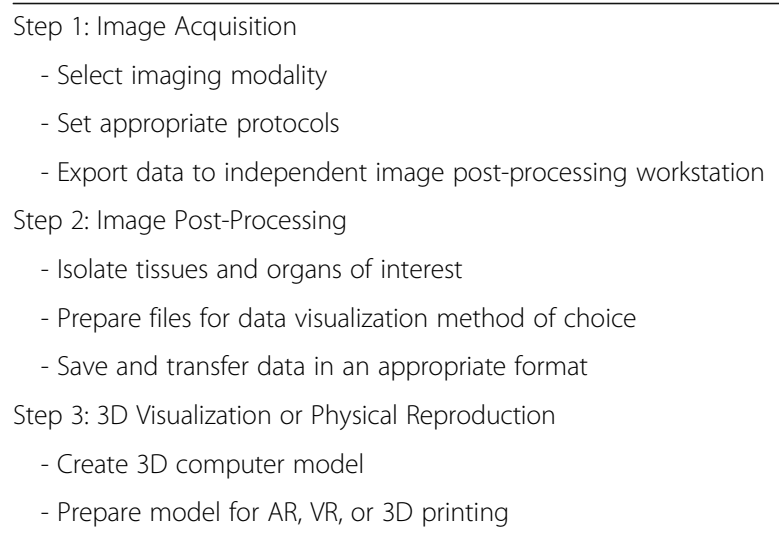


Step 1: Create ROI

Guided Segmentation: Allows us to quickly achieve segmented anatomic ROls

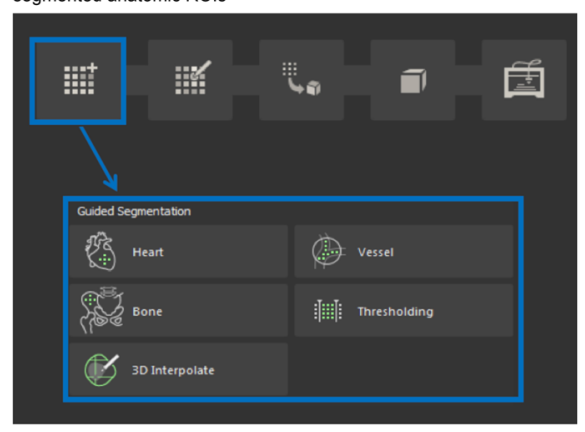

Step 2: Edit RO

Allows us to refine automated segmentation so that we can

acheive anatomically accurate segmentations

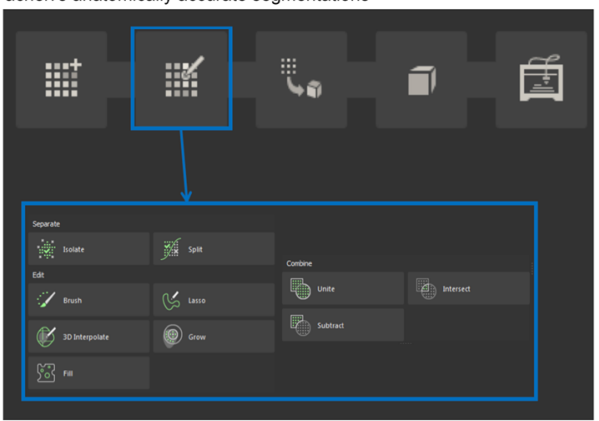

Step 3: Create Part

Creates a 3D part from the segmented ROI

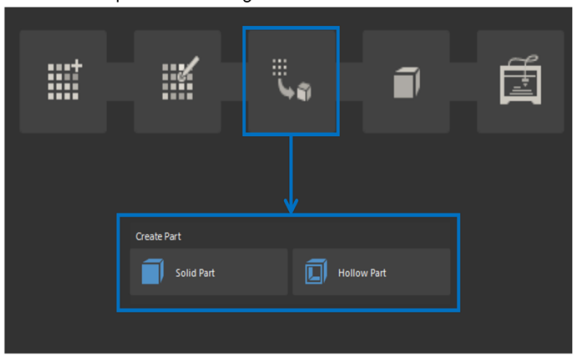

Step 4: Edit Part

Allows us to make minor changes to the part

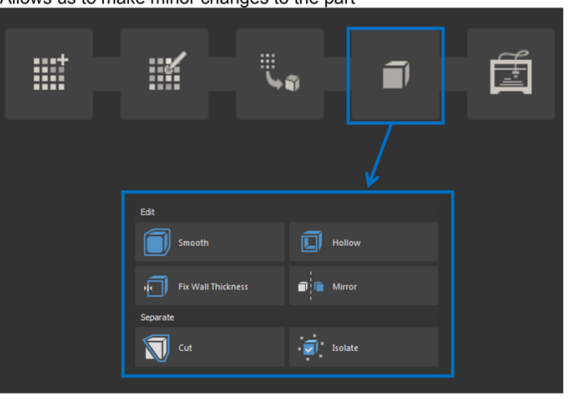

Step 5: Prepare Print

-Allows you to label your 3D model and create connectors between loose

-Allows

- Ensures that the part prepared previously is ready for 3D printing

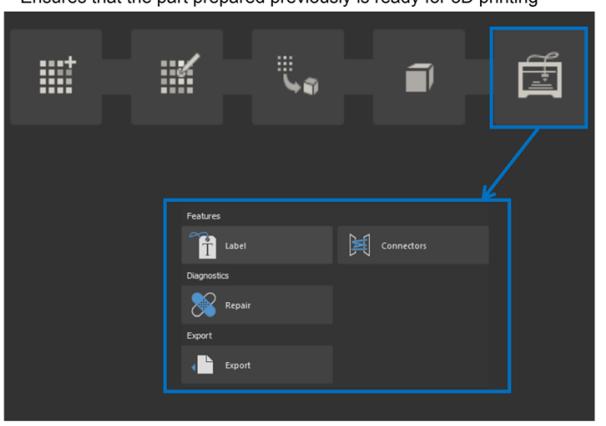

Fig. 1 Mimics InPrint workflow steps including 1) Create ROI, 2) Edit ROI, 3) Add Part, 4) Edit Part, and 5) Prepare Print

a part is the 3D representation of the segmented ROI. The main tools utilized to optimize how images are visualized in Mimics inPrint include zoom, pan, scrolling, zooming, one-click navigation, and threshold adjusting Table 2.

When the software package is opened (Fig. 2a), a DICOM dataset can be loaded by following these steps: click File $\rightarrow$ select New from Disk $\rightarrow$ find folder where DICOMs are stored $\rightarrow$ select the DICOM dataset, making sure that non-strict DICOM is checked $\rightarrow$ select Next to import the images. A window will pop up with the selected study, which allows the study to be verified (patient name, date, number of images, etc.). Once the study is verified, make sure that the study is checked and click "Convert" (Fig. 2b). An orientation window will then appear (Fig. 2c) where the user can ensure the proper orientation is selected. This can be verified with the radiology report of the provider's model request.

The principles of 3D printing in medicine are best understood through practical hands-on experiences covering a broad range of applications. Therefore, this guide will provide the foundational knowledge to broadly cover the segmentation of relevant anatomy on DICOM images followed by 3D printable model creation.

\section{Case 1: Pelvic Fracture}

The pelvis is composed of paired hip bones that are connected in the front at the pubic symphysis and at the 
Table 2 Tools used in Mimics inPrint to optimize the visualization of images

\begin{tabular}{|c|c|c|c|}
\hline Tool & Description & Shortcut 2D Viewport & Shortcut 3D Viewport \\
\hline Zoom & Enlarges the view on the image slice or 3D ROls/Parts & $\begin{array}{l}\text { Right Mouse Button } \\
+ \text { drag }\end{array}$ & $\begin{array}{l}\text { Scroll wheel -or-Ctrl + Right Mouse } \\
\text { Button + drag }\end{array}$ \\
\hline Pan & Planar movement of the image slice or 3D view & \multicolumn{2}{|c|}{ Middle Mouse Button +drag } \\
\hline $\begin{array}{l}\text { Scroll through image } \\
\text { data }\end{array}$ & Jumps to the previous or next slice & Scroll wheel & - \\
\hline Zoom in/out & $\begin{array}{l}\text { Fills the viewport with the entire imaging slice or goes } \\
\text { back to MPR view }\end{array}$ & Spacebar & \\
\hline One-click navigation & Updates the slice position and 3D navigation location & \multicolumn{2}{|c|}{$\begin{array}{l}\text { Left Mouse Button -or-Shift + Left Mouse Button when a tool is } \\
\text { active }\end{array}$} \\
\hline Adjust Threshold & Adjusts contrast window in images & \multicolumn{2}{|c|}{ Ctrl + Right Mouse Button +drag on image } \\
\hline
\end{tabular}

*This table has been modified from inPrint help manual

back by the sacrum. Each hip bone consists of three bones that fuse together during adolescence: the ilium, ischium, and pubis. The ilium, which resembles a butterfly wing, is the largest bone. Below the ilium is a ringshaped structure made up of the ischium and pubis. The acetabulum, a cup-shaped socket that connects with the femoral head to form the hip joint, is the largest movable and weight-bearing joint in the human body.
Pelvic fractures may occur in any location; however due to the complex anatomy of the acetabulum and limited information from plain radiography, the acetabular fracture is the most challenging fracture to manage. 3D printed models can help surgeons to understand the volume, size, and orientation of the bone fragments, allowing them to determine the best reduction technique and surgical approach. 3D printed pelvic models can also

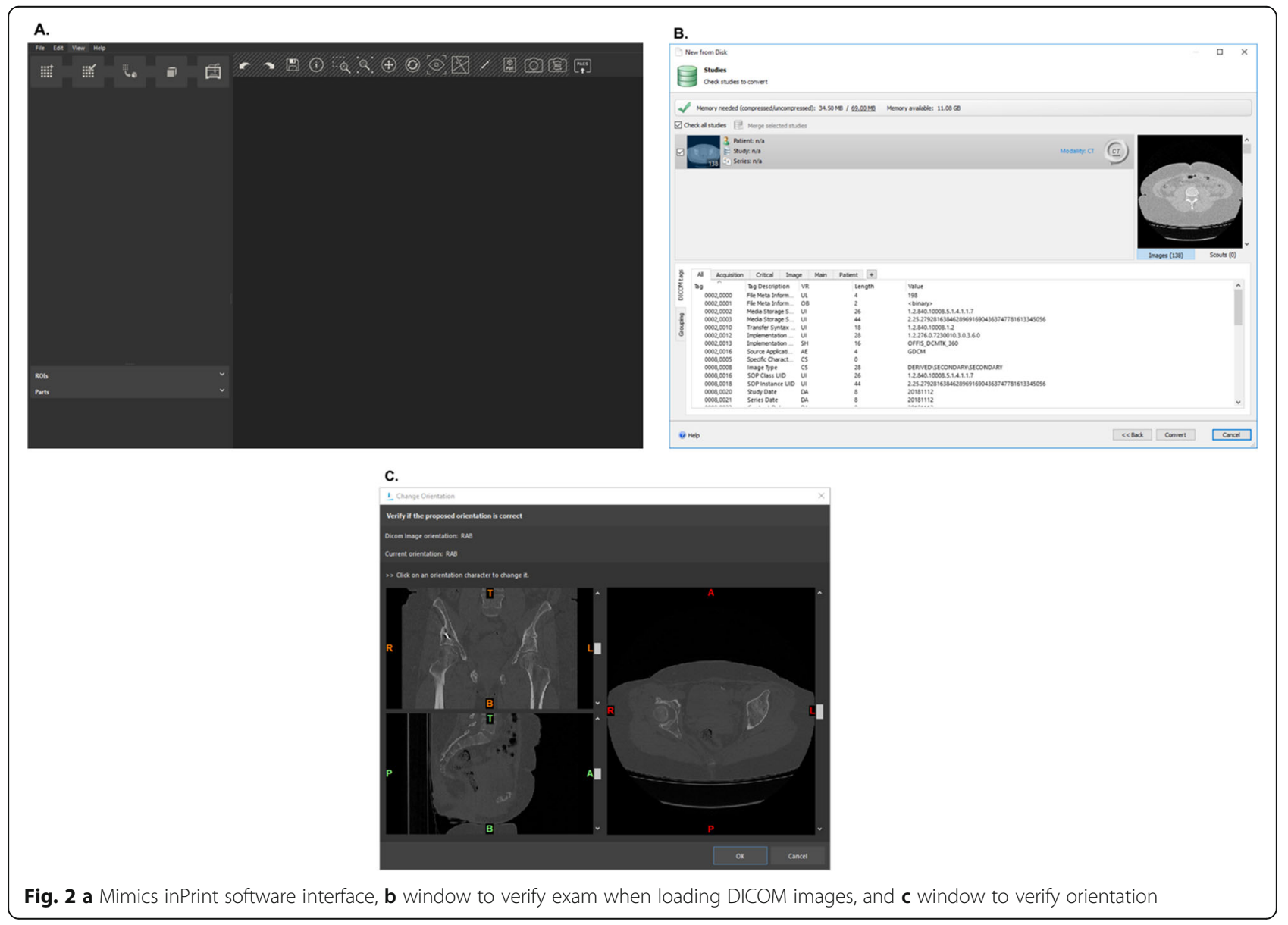


lead to improved perioperative outcomes as compared to patients treated with conventional pre-operative preparation [33]. Mirror images of the opposite intact hemipelvis may also be created and can be used to precontour fixation plates and these have been reported to reduce surgical times [34, 35].

To create a pelvic fracture model, the bony anatomy is segmented from CT DICOM data obtained with a $512 \times$ 512 matrix and $0.781 \mathrm{~mm}$ pixel spacing. For CT images, a good threshold for bone segmentation is between 226 and 3071 Hounsfield Units (HU). Here, the pelvic fracture is on the right side, therefore the bounding box, a box that defines how much of each image is depicted in each window, can be cropped in the coronal, axial, or sagittal viewports to include only the right pelvis (Fig. 3a).

In order to separate the femur from the pelvis, the "Split" tool is utilized. Here, the foreground, the part that we want to keep, is highlighted in blue; and the background, the part that we want to eliminate, is highlighted in gray (Fig. 3b). Painted areas can be drawn in any $2 \mathrm{D}$ viewport and slice. After drawing the pelvis on at least 3 images, clicking the "Validate" button completes the bone segmentation leaving us with just the pelvis portion. Depending on the clinical request, the entire pelvis construct could be prepared for printing or the area could be reduced to just highlight the fractured section. Here we have only included the fractured part in the model. Also, the pubic bone and ischium were divided (Fig. 3c) so that they could be printed using material jetting technology (Stratasys J750, Eden Prairie, MN) in two diferent colors highlighting the fracture (Fig. 3d).
The contralateral side was segmented using the same methods described above and was mirrored onto the fractured side using the "Mirror" tool in order to help guide the surgical procedure. This plan is then exported in 3D portable document format (PDF) for visualization (Additional file 1).

\section{Case 2: Mandible Tumor}

The mandible, the largest of the facial bones, is a single bone connected to the skull by the temporomandibular joint. Malignant tumors of the mandible constitute a diverse group of lesions [36]. 3D printing of mandibular tumors can improve comprehension of anatomy and with the production of cutting guides can enable fast, accurate mandibular reconstructions [37, 38].

In this case, a 52-year-old female presented with an ameloblastoma of the left ramus/mandible. Structures of interest to be included in the 3D printed model include the mandible, tumor, inferior alveolar nerve, and a floating wisdom tooth. The surgey will involve a full-thicknes resection of the mandible in the area of the tumor while attempting to salvage the nerve. Physical simulation of the resection will allow for pre-bending of a titanium reconstruction plate before surgery, potentially saving surgical time and making for a more aesthetic outcome for the patient.

Pre-operative CT images were obtained with the following imaging parameters: $512 \times 512$ matrix, 0.33 $\mathrm{mm}$ pixel spacing, $1 \mathrm{~mm}$ slice thickness, FC80 kernel, and $40 \mathrm{~mA}$.
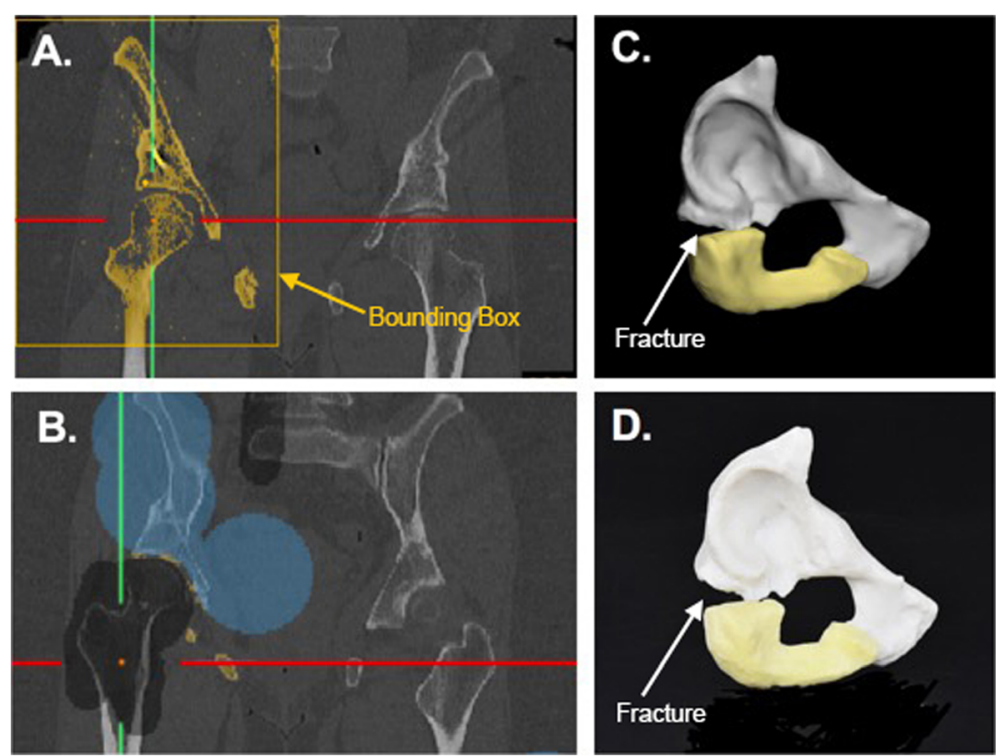

Fig. 3 a Coronal CT image showing threhsolded right pelvic bones, showing similar colors for the pubis, ischium, and femur. b Coronal $C T$ image showing splitting of the pelvis (blue) from the femur (black). c 3D computer model showing the pubis (white) and ischium (yellow). $\mathbf{d}$ Photograph of 3D printed model 


\section{Mandible}

Bone segmentation is performed by setting the threshold between 226 and $3071 \mathrm{HU}$. In this case the bounding box can be cropped in the multi-planar reformat (MPR) view to include only the mandible. Selecting "Keep Largest Region" will ensure that only the largest segment of bone is included.

\section{Tumor}

To segment the tumor, the " $3 D$ interpolate" tool is used in combination with the threshold operation to define the shape of the tumor. The brush tool is used to outline the boundaries of the tumor on diferent slices. The diameter of the brush can be changed using the slide bar or by holding control, left mouse clicking, and dragging. The mode can be changed from "Draw (+)" to "Erase (-)." Here, the minimum and maximum thresholds should be -1024 and 365 respectively.

\section{Nerves}

The nerves can be delineated by manually contouring with 3D interpolation or spline creation (Mimics V22.0, Materialise, Leuven, Belgium).

\section{Teeth}

A preset threshold for "Enamel (CT, adult)" defined as 1553-2850 HU is selected. The bounding box is cropped so that it covers the lower teeth and roots. All the teeth are selected, and manual editing is performed with the
"Brush" tool in erase mode or the "Lasso" tool in the 3D viewport to ensure that the teeth including the floating wisdom tooth are appropriately selected.

The segmented anatomy (Fig. 4a) are converted to 3D parts (Fig. 4b) for better visualization and 3D printing and the 3D anatomy is viewed simultaneously (Fig. 4c). To best depict this anatomy, we chose to print using material jetting (Stratasys J750, Eden Prairie, MN) with the mandible transparent and the tumor and nerves in high presence colors such as blue and green. The total print time for this model was $9 \mathrm{~h}$ and $24 \mathrm{~min}$ using a high mix print setting; and the printed model is shown in Fig. $4 d$.

\section{Case 3: Kidney Tumor}

Over the last 20 years, there has been an increase in the incidence of renal tumors, with renal cell carcinoma (RCC) accounting for approximately $3.5 \%$ of all malignancies $[39,40]$. More complex kidney tumors are associated with longer operative times, warm ischemia times, and greater blood loss [41]. High kidney tumor complexity can also be correlated to the risk of major postoperative complications requiring a secondary intervention [42]. Patient-specific 3D renal tumor models may be used for partial nephrectomy or ablative therapy planning. Having a 3D model can help to assess tumor complexity, as well as the relationship of the tumor to major anatomic structures such as the renal vasculature and
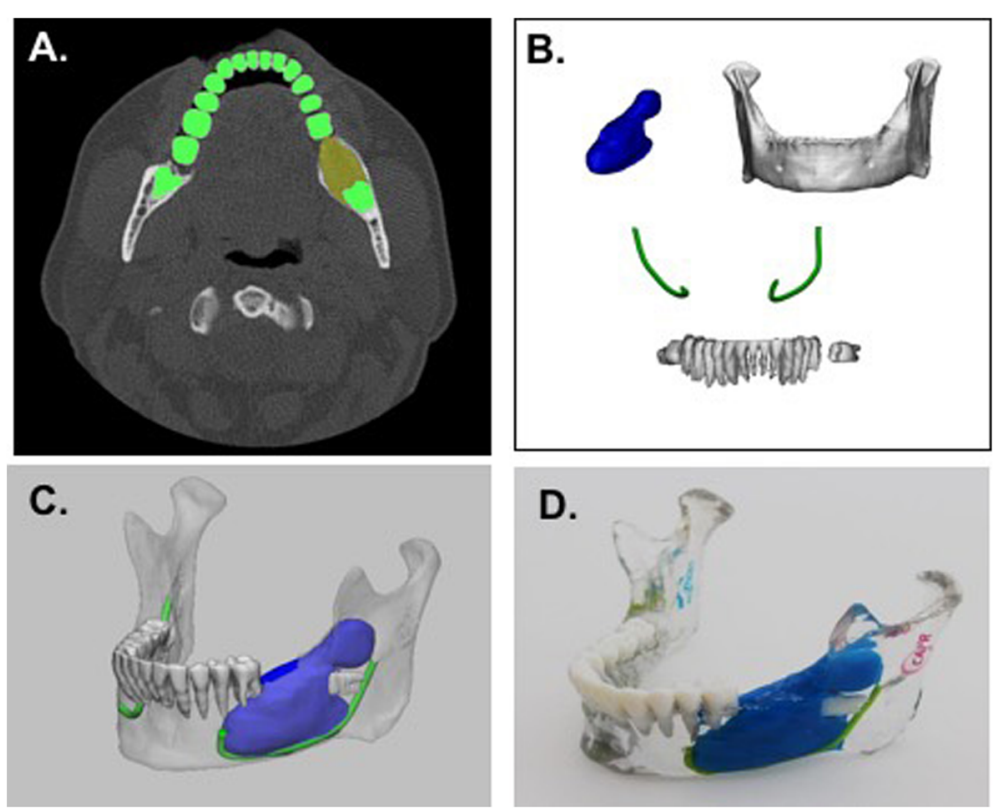

Fig. 4 a Axial CT image showing segmentation of teeth (green) and tumor (yellow). b 3D anatomical regions of interest including the tumor (blue), mandible (white), teeth (white), and nerves (green). c 3D visualization of model including all anatomical parts. $\mathbf{d}$ 3D printed mandible tumor model including the mandible (clear), teeth (white), tumor (blue), and nerves (green) 

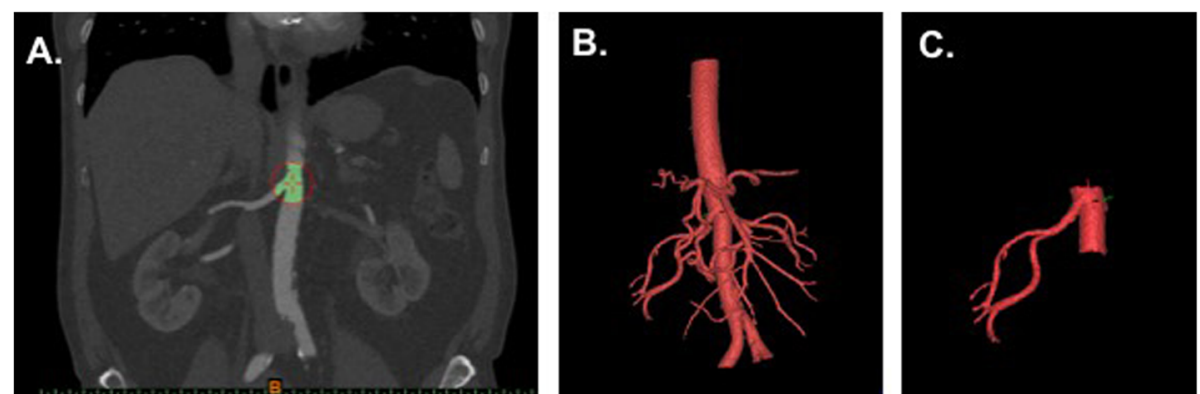

Fig. 5 a Coronal $C T$ image showing aorta and right renal artery selection. b 3D visualization of segmented arterial structures. c Remaining arterial region after trimming has been performed

the renal collecting system [27, 29]. Models may help with improved education of the surgeons allowing for better surgical planning thus possibly reducing warm ischemia and operative times [30].

Here, we present the case of a 72-year-old male with an incidental right renal mass measuring $3.0 \times 2.8 \mathrm{~cm}$, Nephrometry score $=8$ (moderate complexity). The patient decided to undergo robotic assisted partial nephrectomy and a 3D printed model was created to guide the surgical procedure. Pre-operative dual-energy CT images were obtained on a Somatom Force scanner (Siemens, Erlangen, Germany) with the following imaging parameters: $512 \times 512$ matrix, $0.69 \mathrm{~mm}$ pixel spacing, $0.6 \mathrm{~mm}$ slice thickness, $80 \mathrm{kVp}$, Qr44 $\mathrm{d} \backslash 4$ convolution kernel. Isovue 370 contrast (Bracco Diagnotistics Inc., Monroe Township, NJ) was administered intravenously and arterial, venous, and delay phase images were obtained.

\section{Kidney}

To segment the kidney, the "Threshold" tool is used and the "Kidney" preset is selected. For this dataset an optimal threshold value is $60-1000 \mathrm{HU}$. The bounding box is cropped in the orthogonal 2D viewports, the "Keep Largest Region" box is selected, and the "Validate" button is clicked to proceed with the segmentation. Some of the tissue outside the kidney may be selected, so the "Split" tool is used to separate the kidney from the surrounding tissue. The kidney is marked as the foreground and the outside tissue is marked as the background. Once appropriately selected, the "Validate" button is clicked to move forward with the splitting function.

\section{Tumor}

The " $3 D$ Interpolate" tool is utilized to segment the tumor. Here, the "Add" option is utilized instead of the "Threshold" option. The tumor is outlined by drawing with a brush on at least 3 images. Once the tumor is nicely filled in on all of the views, the segmentation can be validated.

\section{Artery}

Using the arterial phase, the "Vessel" tool is used to define the artery. Specifically, the "Blood vessel (CT)" preset is selected and the minimum threshold is adjusted to $300 \mathrm{HU}$. The renal artery is selected and the artery can be grown by left mouse clicking and dragging. There may be additional arteries included in the segmentation that we do not want to include in our final model. The extra vessels may be removed using the "Lasso" tool. Fig. 5 shows the arterial segmentation.
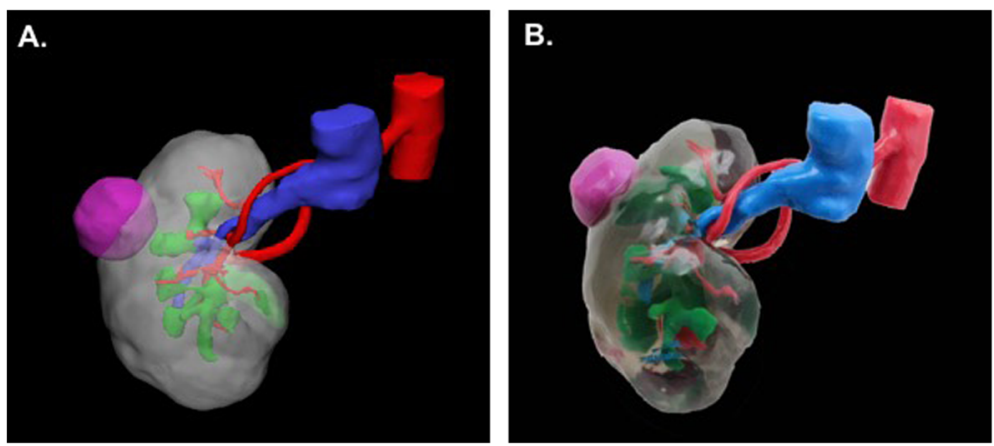

Fig. 6 a 3D visualization of the kidney tumor model and b 3D printed model with the kidney (clear), tumor (purple), renal artery (red), renal vein (blue), and renal collecting system (green) 

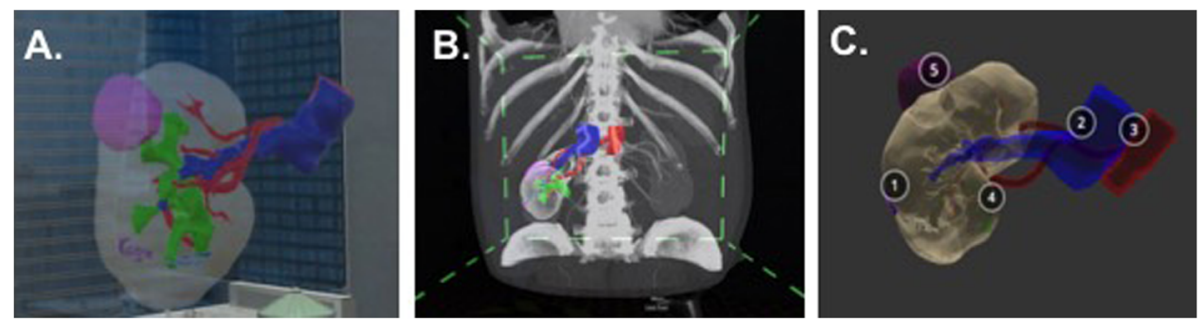

Fig. 7 3D kidney tumor model visualized a in AR using the HoloLens AR headset (Microsoft, Redmond, WA), b in VR using Syglass software (Syglass, IstoVisio, Inc., Morgantown, WV) in combination with the Oculus Rift (Facebook, Menlo Park, CA), and c in VR using the Sketchfab app (Sketchfab, New York, NY) and a smartphone device. Each structure is numbered so that the unfamiliar user can easily identify each individual structure: 1 - kidney, 2 vein, 3 - artery, 4 - collecting system, 5 - renal tumor

\section{Vein and collecting system}

The renal vein and renal collecting system are segmented using the venous and collecting phases respectively. As above, the "Vessel" tool is used to define each region of interest. The vein and collecting system are coregistered to the arterial phase using a point registration method and the segmented anatomical regions of interest are converted to 3D parts.

The segmented anatomy is combined (Fig. 6a) and printing is performed. Here we selected to print using material jetting with the kidney (clear), tumor (purple), renal arteries and aorta (red), renal vein and inferior vena cava (blue), and renal collecting system (green) (Fig. 6b).

For all cases described above, an AR or VR model may be created from the segmented datasets. For preparation, each individual part can be exported in alias wavefront (.obj) format or each model including all of the parts can be exported in .vrml format. Models can be prepared in Unity, a cross-platform game engine (Unity Technologies, San Francisco, CA), for deployment in the AR headset [43] or can be visualized using a VR headset or cellular device (Fig. 7). The workflow for creating AR models in Unity has been previously described [44] and requires setting up a virtual camera and placing the 3D content a certain distance away for visualization.

\section{Conclusion}

Converting DICOM data to printable formats is a complex process requiring multiple steps. This paper describes key steps to create 3D printed CMF, orthopedic, and renal models. Techniques described here may also be applied to other organs and anatomical regions of interest. The number of 3D printed and AR/VR models generated from DICOM images is growing exponentially at the point of care. It is essential that radiologists and other health care professionals understand this complex process.

\section{Supplementary information}

Supplementary information accompanies this paper at https://doi.org/10. 1186/s41205-019-0054-y.

Additional file 1. Figure S1: 3D PDF of the kidney tumor model. Figure S2: 3D PDF of the mandible tumor model. Figure S3: 3D PDF of the pelvic fracture model.

\section{Abbreviations}

3D: Three-dimensional; AR: Augmented reality; CMF: Cranio-maxillofacial; CT: Computed tomography; DICOM: Digital imaging and communications in medicine; FDA: Food and Drug Administration; HU: Hounsfield unit; MPR: Multi-planar reformat; MRI: Magentic resonance imaging; OBJ: Wavefront object file; PDF: Portable document format; RCC: Renal cell carcinoma; ROI: Region of interest; RSNA: Radiological Society of North America; SIG: Special Interest Group; STL: Stereolithography, standard tesselation language, or standard triangle language; VR: Virtual reality

\section{Acknowledgements}

The corresponding author (NW) receives in-kind support from Stratasys and is a consultant for GE Healthcare. This work was supported in part by NIH P41 EB17183.

The article processing charge for this publication has been funded generously by an unrestricted educational grant from Stratasys.

\section{Authors' contributions}

NW - Course design, imaging, 3D image post processing and modeling, manuscript writing, manuscript editing. AEA - Course design, 3D image post processing and modeling, manuscript editing. AMC - Course design, 3D image post processing and modeling, manuscript editing. PCL - Course design, 3D image post processing and modeling, manuscript editing. MS Course design, 3D image post processing and modeling, manuscript editing. TP - Course design, 3D image post processing and modeling, manuscript editing. JM - Course design, 3D image post processing and modeling, manuscript editing. All authors read and approved the final manuscript.

Funding

Grant Support: NIH P41 EB017183.

In-Kind Research Support: Stratasys.

Availability of data and materials

The datasets used and/or analyzed during the current study are available from the corresponding author on reasonable request. We also plan to create a public link the the DICOM files, so readers can access the DICOM data used to create these models.

Ethics approval and consent to participate N/A 


\section{Consent for publication}

All authors have approved the manuscript for submission.

\section{Competing interests}

Financial and Non-Financial Disclosures related to this work:

NW -In-Kind Research Support: Stratasys, Ltd.

TP and MS are employees of Materialise USA

Financial and Non-Financial Disclosures not related to this work:

NW - Consultant, GE Healthcare.

No competing interests: $A A, A M C, P L, J M$

One of the authors (NW) has in-kind research support from Stratasys. None

of the remaining authors have any relationship with Stratasys.

\section{Author details}

${ }^{1}$ Department of Radiology, Montefiore Medical Center, Albert Einstein College of Medicine, 111 East 210th Street, Bronx, NY 10467, USA. ${ }^{2}$ Center for Advanced Imaging Innovation and Research (CAI2R) and Bernard and Irene Schwartz Center for Biomedical Imaging, Department of Radiology, NYU Langone Health, NYU School of Medicine, New York, NY, USA. ${ }^{3}$ Department of Radiology, Mayo Clinic, Rochester, MN, USA. ${ }^{4}$ Department of Radiology, The Ottawa Hospital, Ottawa, Canada. ${ }^{5}$ 3D Medical Applications Center, Walter Reed National Military Medical Center, Bethesda, MD, USA. ${ }^{6}$ Materialise USA, Plymouth, MI, USA

Received: 18 July 2019 Accepted: 13 December 2019 Published online: 30 December 2019

\section{References}

1. Lipson HKM. Fabricated: the new world of 3D printing. Indianapolis: Wiley; 2013.

2. Mitsouras D, Liacouras $P$, Imanzadeh $A$, et al. Medical 3D printing for the radiologist. Radiographics. 2015;35:1965-88.

3. Ventola CL. Medical applications for 3D printing: current and projected uses. P T. 2014;39:704-11.

4. Randall DMP, Dev Bardhan K, Spencer P, Gillott R, de Noronha R, Fenner JW. The oculus rift virtual colonoscopy: introducing a new technology and initial impressions. J Biomed Graph Comp. 2016:6:34-42.

5. Sutherland J, Belec J, Sheikh A, et al. Applying modern virtual and augmented reality technologies to medical images and models. J Digit Imaging. 2019;32:38-53.

6. Giannopoulos AA, Chepelev L, Sheikh A, et al. 3D printed ventricular septal defect patch: a primer for the 2015 Radiological Society of North America (RSNA) hands-on course in 3D printing. 3D Print Med. 2015;1:3.

7. Chepelev L, Hodgdon T, Gupta A, Wang A, Torres C, Krishna S, Akyuz A, Mitsouras D, Sheikh A. Medical 3D printing for vascular interventions and surgical oncology: a primer for the 2016 radiological society of North America (RSNA) hands-on course in 3D printing. 3D Print Med. 2016;2:5.

8. Chepelev L, Souza C, Althobaity W, et al. Preoperative planning and tracheal stent design in thoracic surgery: a primer for the 2017 Radiological Society of North America (RSNA) hands-on course in 3D printing. 3D Print Med. 2017;3:14

9. Di Prima M, Coburn J, Hwang D, et al. 3D Print Med. 2016;2:1. https://doi. org/10.1186/s41205-016-0005-9

10. Materialise Mimics inPrint: Regulatory Information. https://www.materialise. com/en/medical/software/materialise-mimics-inprint/regulatory-information. Accessed 18 April 2019.

11. FDA's Role in 3D Printing. https://www.fdagov/medical-devices/3d-printingmedical-devices/fdas-role-3d-printing 2017; Accessed October 28, 2019.

12. Toth BA, Ellis DS, Stewart WB. Computer-designed prostheses for Orbitocranial reconstruction. Plast Reconstr Surg. 1988;81:315-22.

13. Scholz $M$, Eufinger $H$, Wehmoller $M$, et al. CAD/CAM (computer-aided design/computer-aided manufacturing) titanium implants for cranial and craniofacial defect reconstruction. Zentralbl Neurochir. 1997:58:105-10.

14. Klein HM, Schneider W, Alzen G, et al. Pediatric craniofacial surgery: comparison of milling and stereolithography for 3D model manufacturing. Pediatr Radiol. 1992:22:458-60.

15. Andrews BT, Thurston TE, Tanna N, et al. A multicenter experience with image-guided surgical navigation: broadening clinical indications in complex Craniomaxillofacial surgery. J Craniofac Surg. 2015;26:1136-9.

16. Bolzoni Villaret A, Battaglia P, Tschabitscher M, et al. A 3-dimensional transnasal endoscopic journey through the paranasal sinuses and adjacent skull base: a practical and surgery-oriented perspective. Neurosurgery. 2014; 10(Suppl 1):116-20 discussion 20.

17. Dehdashti AR, Ganna A, Witterick I, Gentili F. Expanded endoscopic endonasal approach for anterior cranial base and suprasellar lesions: indications and limitations. Neurosurgery. 2009;64:677-87 discussion 87-9.

18. Levine JP, Patel A, Saadeh PB, Hirsch DL. Computer-aided design and manufacturing in craniomaxillofacial surgery: the new state of the art. J Craniofac Surg. 2012;23:288-93.

19. Levine JP, Bae JS, Soares M, et al. Jaw in a day: total maxillofacial reconstruction using digital technology. Plast Reconstr Surg. 2013;131:1386-91.

20. Hirsch DL, Garfein ES, Christensen AM, et al. Use of computer-aided design and computer-aided manufacturing to produce orthognathically ideal surgical outcomes: a paradigm shift in head and neck reconstruction. J Oral Maxillofac Surg. 2009;67:2115-22.

21. Potamianos $\mathrm{P}, \mathrm{Amis} \mathrm{AA}$, Forester $\mathrm{AJ}$, et al. Rapid prototyping for orthopaedic surgery. Proc Inst Mech Eng H. 1998;212:383-93.

22. Shuang F, Hu W, Shao Y, et al. Treatment of Intercondylar humeral fractures with 3D-printed Osteosynthesis plates. Medicine (Baltimore). 2016;95:e2461.

23. Kim D, Lim JY, Shim KW, et al. Sacral reconstruction with a 3D-printed implant after Hemisacrectomy in a patient with sacral osteosarcoma: 1-year follow-up result. Yonsei Med J. 2017:58:453-7.

24. Hamid KS, Parekh SG, Adams SB. Salvage of severe foot and ankle trauma with a 3D printed scaffold. Foot Ankle Int. 2016:37:433-9.

25. Chana-Rodriguez F, Mananes RP, Rojo-Manaute J, et al. 3D surgical printing and pre contoured plates for acetabular fractures. Injury. 2016;47:2507-11.

26. Ma L, Zhou Y, Zhu Y, et al. 3D-printed guiding templates for improved osteosarcoma resection. Sci Rep. 2016:6:23335.

27. Silberstein $J$, Maddox MM, Dorsey $P$, et al. Physical models of renal malignancies using standard cross-sectional imaging and 3-dimensional printers: a pilot study. Urology. 2014;84:268-72.

28. Bernhard JC, Isotani S, Matsugasumi T, et al. Personalized 3D printed model of kidney and tumor anatomy: a useful tool for patient education. World $J$ Urol. 2016;34:337-45

29. Wake N, Chandarana H, Huang WC, et al. Application of anatomically accurate, patient-specific 3D printed models from MRI data in urological oncology. Clin Radiol. 2016;71:610-4.

30. Wake N, Rude T, Kang SK, et al. 3D printed renal cancer models derived from MRI data: application in pre-surgical planning. Abdom Radiol (NY). 2017:42:1501-9.

31. Chepelev L, Wake N, AW RJ, Gupta A, Arribas E, Santiago L, Ballard D, Wang KC, Weadock W, Ionita CN, Mitsouras D, Morris J, Matsumoto J, Christensen A, Liacouras P, Rybicki FJ, Sheikh A, RSNA Special Interest Group for 3D Printing. Radiological Society of North America (RSNA) 3D Printing Special Interest Group (SIG): guidelines for medical 3D printing and appropriateness for clinical scenarios. 3D Print Med. 2018:4:1-38.

32. Mottart $X$, Slagmolen P. 3D Lab in a Hospital Environment: 3 key reasons why a professional desktop printer is a great fit. Materialise NV White Paper. 2017:1:1-19

33. Li B, Chen B, Zhang Y, et al. Comparative use of the computer-aided angiography and rapid prototyping technology versus conventional imaging in the management of the tile C pelvic fractures. Int Orthop. 2016:40:161-6.

34. Hung CC, Li YT, Chou YC, et al. Conventional plate fixation method versus pre-operative virtual simulation and three-dimensional printing-assisted contoured plate fixation method in the treatment of anterior pelvic ring fracture. Int Orthop. 2019:43:425-31.

35. Li YT, Hung CC, Chou YC, et al. Surgical treatment for posterior dislocation of hip combined with Acetabular fractures using preoperative virtual simulation and three-dimensional printing model-assisted Precontoured plate fixation techniques. Biomed Res Int. 2019;2019:3971571.

36. Neville BWDD, Allen CM, Bouquot JE. Oral and Maxillofacial Pathology; 1995

37. Chutchurru MS, Brusca MI, Palavecino R. Ameloblastoma: reconstruction using titanium plates adapted using Stereolithographic models. Ann Maxillofac Surg. 2018;8:337-41.

38. Dupret-Bories A, Vergez S, Meresse T, et al. Contribution of 3D printing to mandibular reconstruction after cancer. Eur Ann Otorhinolaryngol Head Neck Dis. 2018;135:133-6.

39. Chen N, Zhu K, Wang C, Sun X. 3D printing technique for guiding complicated cardiovascular surgery. J Thorac Dis. 2017;9:E945-E6.

40. Sivarajan G, Huang WC. Current practice patterns in the surgical management of renal cancer in the United States. Urol Clin North Am. 2012; 39:149-60. 
41. Ellison JS, Montgomery JS, Hafez KS, et al. Association of RENAL nephrometry score with outcomes of minimally invasive partial nephrectomy. Int J Urol. 2013;20:564-70.

42. Simhan J, Smaldone MC, Tsai KJ, et al. Objective measures of renal mass anatomic complexity predict rates of major complications following partial nephrectomy. Eur Urol. 2011;60:724-30.

43. Wake N, Rosenkrantz AB, Huang R, et al. Patient-specific $3 D$ printed and augmented reality kidney and prostate cancer models: impact on patient education. 3D Print Med. 2019;5:4.

44. Wake N, Bjurlin MA, Rostami P, et al. Three-dimensional printing and augmented reality: enhanced precision for robotic assisted partial nephrectomy. Urology. 2018;116:227-8.

\section{Publisher's Note}

Springer Nature remains neutral with regard to jurisdictional claims in published maps and institutional affiliations.

Ready to submit your research? Choose BMC and benefit from:

- fast, convenient online submission

- thorough peer review by experienced researchers in your field

- rapid publication on acceptance

- support for research data, including large and complex data types

- gold Open Access which fosters wider collaboration and increased citations

- maximum visibility for your research: over $100 \mathrm{M}$ website views per year

At $\mathrm{BMC}$, research is always in progress.

Learn more biomedcentral.com/submissions 